\title{
Chaos prediction in nano-resonators based on nonlocal elasticity theory
}

\author{
Hassan Nahvi, ${ }^{1 *}$ and Mehdi Maleki ${ }^{1}$ \\ ${ }^{1}$ Department of Mechanical Engineering, Isfahan University of Technology, Isfahan 8415683111, Iran
}

\begin{abstract}
By decreasing the thickness of micro- and nano- beams, classical continuum theory is not accurate to predict the static and dynamic response due to the absence of length scale parameter. In this paper, nonlocal elasticity theory is used to detect chaos in nano-resonators. In this way, first mode shape of the nano-beam is found and Galerkin method is used to convert the governing partial differential equation to an ordinary differential equation. Melnikov method is used to determine the critical value of AC actuation voltage resulting chaotic motion. Effects of nonlocal parameter and beam thickness on the stability region of the resonator are investigated. It will be shown that increasing the nonlocal parameter and decreasing the beam thickness increases the difference between stability regions obtained by classical and nonlocal theories. Moreover, increasing the nonlocal parameter decreases the nonlinear stiffness and increases the critical actuation voltage which may lead to chaotic motion.
\end{abstract}

\section{Introduction}

Several decades have passed by since the discovery and development of micro-electro-mechanical systems (MEMS). One important type of MEMS is resonator which has various applications [1]. Due to nonlinear behavior of this device caused by nonlinear electrical force, squeezing film damping, mid-plane stretching and other geometrical nonlinearities, some complicated phenomena have been seen in the dynamic behavior of resonators. Nonlinearities also may lead to chaotic behavior [2]. Haghighi and Markazi [3] used Melnikov analysis method to predict chaos in MEMS resonators. They proposed an analytical criterion for homoclinic chaos in the form of an inequality which is written in terms of the system parameters.

Experimental tests have shown that static and dynamic behavior of micro- and nano- beams cannot be predicted by classical continuum theory with the absence of an intrinsic length scale in the constitutive relations [4]. Nonlocal elasticity theory is one of the various higher-order elasticity theories which have been used to develop microstructure-dependent beam models in order to study the static and dynamic response of micro- and nano-beams $[5,6]$.

Many researches on the local and nonlocal theories have been carried out not only considering the beam model but also considering the thin shell model, in the linear and nonlinear fields, with particular application to carbon nanotubes $[7,8]$.

In this paper, based on Melnikov analysis method, chaotic region for a nano-resonator obtained with nonlocal elasticity theory will be compared with that of classical one and the effect of nonlocal parameter on the safe region of the resonator in which the system has harmonic response will be investigated.

\section{Mathematical modeling}

Figure 1 shows layout of a conventional resonator under electrical actuation.

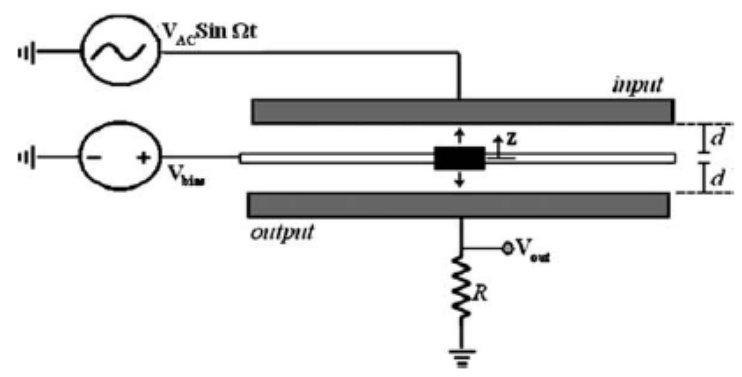

Figure 1. Layout of a nano-resonator under electrical actuation [3].

Dynamic equation of the nano-resonator based on nonlocal theory can be written as [6]:

$$
\begin{aligned}
& -2 E I\left(e_{0} a\right)^{2} \frac{\partial^{6} w}{\partial x^{6}}+\left(E A+\left(e_{0} a\right)^{2} P\right) \frac{\partial^{4} w}{\partial x^{4}} \\
& +\rho A \frac{\partial^{2} w}{\partial t^{2}}+C \frac{\partial w}{\partial t}-\left(P+\frac{E A}{2 L} \int_{0}^{1}\left(\frac{\partial w}{\partial x}\right)^{2} d x\right) \frac{\partial^{2} w}{\partial x^{2}}-q(x, t)=0
\end{aligned}
$$

where $w$ is beam deflection, $E I$ is flexural rigidity, $A$ is beam cross-sectional area, $\rho$ is material mass density, $P$ is axial force, $C$ is damping coefficient,

\footnotetext{
* Corresponding author: hnahvi@cc.iut.ac.ir
} 
$q$ is external load and $e_{0} a$ is the size effect parameter which can be obtained by fitting against experiments and molecular dynamic simulations. The terms appear in Eq. (1) correspond to the size effect (which is ignored in the classical Euler-Bernoulli beam model), micro-beam elastic force, micro-beam acceleration, damping, axial force and mid-plane stretching, and external load, respectively.

Using the following non-dimensional parameters, Eq. (1) reduces to Eq. (3) as:

$$
\begin{gathered}
\bar{x}=\frac{x}{L}, \quad \bar{w}=\frac{w}{g}, \quad \bar{t}=\frac{t}{T} \\
\mathfrak{R}(\bar{w})=-2 \tau_{n}^{2} \frac{\partial^{6} \bar{w}}{\partial \bar{x}^{6}}+\left(1+\bar{P} \tau_{n}^{2}\right) \frac{\partial^{4} \bar{w}}{\partial \bar{x}^{4}}+\frac{\partial^{2} \bar{w}}{\partial \bar{t}^{2}}+c \frac{\partial \bar{w}}{\partial \bar{t}} \\
-\left(\bar{P}+\alpha \int_{0}^{1}\left(\frac{\partial \bar{w}}{\partial \bar{x}}\right)^{2} d \bar{x}\right) \frac{\partial^{2} \bar{w}}{\partial \bar{x}^{2}}-\bar{q}=0
\end{gathered}
$$

where $L$ is the micro-beam length and $g$ is the gap between two electrodes and

$$
\begin{aligned}
& \alpha_{1}=\frac{E A g^{2}}{2 E I}, \quad \bar{P}=\frac{L^{2}}{E I} P, \tau_{n}=\frac{e_{0} a}{L}, \\
& c=\frac{C L^{4}}{E I T}, T=\sqrt{\frac{\rho b h L^{4}}{E I}}
\end{aligned}
$$

In order to use Galekin method, linear mode shapes of beam based on nonlocal theory should be found. The solution of Eq. (3) can be assumed as [9]:

$$
\bar{w}_{n}(\bar{x}, \bar{t})=\psi_{n}(\bar{x}) \sin \left(\omega_{n} \bar{t}\right)
$$

where $\psi_{n}(\bar{x})$ is the nth mode function and $\omega_{n}$ is the dimensionless natural frequency. The natural frequencies and mode shapes of the beam are found by setting nonlinear terms and external excitation in Eq. (3) equal to zero. Substituting Eq. (5) into Eq. (3) yields:

$$
-2 \tau_{n}^{2} \frac{\mathrm{d}^{6} \psi_{n}}{\mathrm{~d} \bar{x}^{6}}+\left(1+\bar{P} \tau_{n}^{2}\right) \frac{\mathrm{d}^{4} \psi_{n}}{\mathrm{~d} \bar{x}^{4}}-\omega_{n}{ }^{2} \psi_{n}=0
$$

Micro-beam boundary conditions for clamped ends based on the nonlocal theory are [7]:

$$
\begin{aligned}
& \psi_{n}(0)=\psi_{n}(1)=0 \\
& \frac{d \psi_{n}(0)}{d \bar{x}}=\frac{d \psi_{n}(1)}{d \bar{x}}=0 \\
& \frac{d^{3} \psi_{n}(0)}{d \bar{x}^{3}}+3 \tau_{n}^{2} \frac{d^{5} \psi_{n}(0)}{d \bar{x}^{5}}=\frac{d^{3} \psi_{n}(1)}{d \bar{x}^{3}}+3 \tau_{n}^{2} \frac{d^{5} \psi_{n}(1)}{d \bar{x}^{5}}=0
\end{aligned}
$$

General solution of Eq. (6) can be written as:

$$
\begin{aligned}
& \psi_{n}(\bar{x})=C_{1} e^{\beta_{1} \bar{x}}+C_{2} e^{\beta_{2} \bar{x}}+C_{3} e^{\beta_{3} \bar{x}}+C_{4} e^{\beta_{4} \bar{x}} \\
& +C_{5} e^{\beta_{5} \bar{x}}+C_{6} e^{\beta_{6} \bar{x}}=C_{k} \sum_{k=1}^{6} e^{\beta_{k} \bar{x}}
\end{aligned}
$$

In the above equation, $C_{1}$ to $C_{6}$ are integration constants of the sixth-order differential equation, Eq. (6), and $\beta_{1}$ to $\beta_{6}$ are the roots of the following equation:

$$
-2 \tau_{n}^{2} \beta^{6}+\left(1+\bar{P} \tau_{n}^{2}\right) \beta^{4}-\omega_{n}^{2}=0
$$

Substituting Eq. (8) into Eq. (7) gives the following matrix equation:

$$
\begin{aligned}
& \mathbf{A}\left(\beta_{1}, \beta_{2}, \beta_{3}, \beta_{4}, \beta_{5}, \beta_{6}\right)\left[C_{1}, C_{2}, C_{3}, C_{4}, C_{5}, C_{6}\right]^{T} \\
& =\mathbf{0}
\end{aligned}
$$

To obtain non-trivial solution for the constants $C_{i}$, the determinant of matrix A should be zero, thus:

$$
|\mathbf{A}|=0
$$

By using Eqs. (9) and (11), the seven unknown quantities $\left(\beta_{1}, \beta_{2}, \beta_{3}, \beta_{4}, \beta_{5}, \beta_{6}, \omega_{n}\right)$ can be found. Substituting the results into Eq. (10) and setting $C_{1}$ as an arbitrary constant gives the constants $C_{2}$ to $C_{6}$. The one-parameter Galerkin's solution may be determined using the following relation:

$$
\int_{0}^{1} \mathfrak{R}(\overline{\mathrm{w}}) \psi_{n}(\bar{x}) d \bar{x}=0
$$

where $\overline{\mathrm{w}}=\psi_{n}(\bar{x}) z(\bar{t})$ based on the variable separation and $z$ is the beam mid-point deflection.

The governing differential equation of the beam midpoint, $z(\bar{t})$, is found to be:

$$
m z^{\prime \prime}+k_{1} z+k_{3} z^{3}+b z^{\prime}=F_{\text {act }}
$$

where $\left({ }^{\prime}\right)$ represents derivative with respect to $\bar{t}$. The parameters in Eq. (13) are:

$$
m=\int_{0}^{1} \psi_{n}(\bar{x})^{2} d \bar{x}
$$

$$
\begin{aligned}
& k_{1}= \\
& \int_{0}^{1} \psi_{n}(\bar{x})\left(-2 \tau_{n}^{2} \frac{\mathrm{d}^{6} \psi_{n}(\bar{x})}{\mathrm{d} \bar{x}^{6}}+\left(1+\bar{P} \tau_{n}^{2}\right) \frac{\mathrm{d}^{4} \psi_{n}(\bar{x})}{\mathrm{d} \bar{x}^{4}}-\bar{P} \frac{\mathrm{d}^{2} \psi_{n}(\bar{x})}{\mathrm{d} \bar{x}^{2}}\right) \mathrm{d} \bar{x} \\
& k_{3}=-\int_{0}^{1} \alpha_{1} \psi_{n}(\bar{x}) \frac{\mathrm{d}^{2} \psi_{n}(\bar{x})}{\mathrm{d} \bar{x}^{2}}\left(\int_{0}^{1}\left(\frac{\mathrm{d} \psi_{n}(\bar{x})}{\mathrm{d} \bar{x}}\right)^{2} \mathrm{~d} \bar{x}\right) \mathrm{d} \bar{x} \\
& b=c \int_{0}^{1} \psi_{n}(\bar{x})^{2} d \bar{x}
\end{aligned}
$$


In Eq. (13), $F_{\text {act }}$ is the electrostatic force which is the combination of $\mathrm{DC}$ and $\mathrm{AC}$ actuations. By introducing non-dimensional time as $\tau=\omega_{0} \bar{t}$, Eq. (13) can be rewritten as:

$$
\begin{aligned}
& \ddot{z}+z+\beta z^{3}+\mu \dot{z}= \\
& \gamma\left(\frac{1}{(1-z)^{2}}-\frac{1}{(1+z)^{2}}\right)-\frac{A \cos (\omega \tau)}{(1-z)^{2}}
\end{aligned}
$$

where $(\cdot)$ denotes derivation with respect to $\tau$. The parameters in Eq. (15) are defined as:

$$
\begin{aligned}
& \omega_{0}=\sqrt{\frac{k_{1}}{m}} ; \gamma=\frac{\varepsilon b l^{4} V_{b}^{2}}{2 \omega_{0}^{2} E I g^{3}}, \quad A=2 \gamma \frac{V_{A C}}{V_{b}}, \\
& \beta=\frac{k_{3}}{m \omega_{0}^{2}}, \quad \mu=\frac{b}{m \omega_{0}}, \omega=\frac{T \Omega}{\omega_{0}}
\end{aligned}
$$

In Eq. (16), $\varepsilon$ is the permittivity of vacuum, $V_{b}$ is the bias DC voltage, $V_{A C}$ is the amplitude of applied AC voltage and as shown in Figure (1), $\Omega$ is the frequency of AC voltage.

\section{Melnikov analysis}

In order to apply Melnikov method, Taylor expansion is applied to Eq. (15) and then is written in state space form as [3]:

$$
\begin{aligned}
& \dot{z}=y \\
& \dot{y}=(1-4 \gamma) z+(\beta-8 \gamma) z^{3} \\
& +\varepsilon\left(-\bar{\mu} \dot{z}+\frac{\bar{A}}{(1-z)^{2}} \operatorname{Sin} \omega \tau\right)
\end{aligned}
$$

where $\mu \equiv \varepsilon \bar{\mu}$ and $A \equiv \varepsilon \bar{A}$ (i.e., $\mu=O(\varepsilon), A=O(\varepsilon)$ ) and $\varepsilon$ is a small parameter. These assumptions are valid for a MEMS resonator with high quality factor and $\mathrm{AC}$ diving voltage much smaller than the bias voltage. The Melnikov function is proportional to the first variation of the distance function between stable and unstable manifolds. The Melnikov function of Eq. (17) is:

$$
M\left(\tau_{0}\right)=\int_{-\infty}^{\infty}\left[y_{0}\left(-\bar{\mu} y_{0}+\frac{\bar{A}}{\left(1-z_{0}\right)^{2}} \operatorname{Sin} \omega\left(\tau+\tau_{0}\right)\right)\right] d \tau
$$

where $\left(z_{0}, y_{0}\right)$ is the solution corresponding to the unperturbed homoclinic trajectory, as

$$
\begin{aligned}
& \dot{z}=y \\
& \dot{y}=(1-4 \gamma) z+(\beta-8 \gamma) z^{3}
\end{aligned}
$$

The solution of Eq. (19) is analytically obtained as:

$$
\begin{aligned}
& \left(z_{0}, y_{0}\right)=\mp z_{e}(\operatorname{sech}(\sqrt{1-4 \gamma} \tau), \\
& \operatorname{sech}(\sqrt{1-4 \gamma} \tau) \tanh (\sqrt{1-4 \gamma} \tau))
\end{aligned}
$$

where $z_{e}$ is found by setting potential function equal to zero.

The integral in Eq. (18) is solved using residue theorem and the following inequality was found for the chaos region [3]:

$$
\frac{\bar{A}}{\bar{\mu}} \geq\left|\frac{z_{e} \sqrt{4 \gamma-1} \sqrt{1-z_{e}^{2}} \operatorname{Sinh}[\bar{\omega} \pi]}{3 \pi \bar{\omega} \operatorname{Sinh}\left[-\bar{\omega}\left(\operatorname{ArcCos}\left[z_{e}\right]\right)\right]}\right|
$$

where $\bar{\omega}=\frac{\omega}{\sqrt{4 \gamma-1}}$. For more details the reader is referred to the reference [3].

\section{Simulations and results}

In this section, the Melnikov method will be used to analyse the effect of the size on the chaotic motion of nano-resonators. In the following, a nano-beam made of silicon will be considered as an illustrative example. From experimental and numerical atomic analyses, the relevant bulk material properties for silicon are: $E=165 \mathrm{GPa}, \quad v=0.24$ and $\rho=2332 \mathrm{~kg} / \mathrm{m}^{3}$. The nano-resonator geometrical parameters used here are listed in Table 1.

Table 1. Nano-resonator parameters.

\begin{tabular}{c|c}
\hline Parameter & Value \\
\hline Length & $L=50 \mu \mathrm{m}$ \\
\hline Width & $b=5 \mu \mathrm{m}$ \\
\hline Thickness & $h=1 \mu \mathrm{m}$ \\
\hline Initial gap & $g=3 \mu \mathrm{m}$ \\
\hline
\end{tabular}

Figure 2 shows the effect of nonlocal parameter on the chaotic motion of the nano-resonator using Eq. (21). It should be noted that the region below the curve corresponds to the harmonic motion and chaotic motion will not occur in this region. As shown, for small values of the nonlocal parameter, both theories lead to the same result but increasing the nonlocal parameter increases the difference between the results obtained by the two theories. 


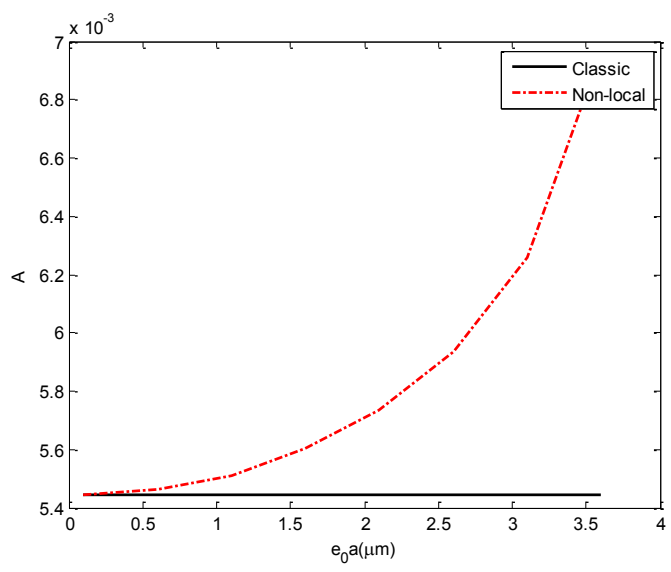

Figure 2. Critical values for $\mathrm{AC}$ as a function of nonlocal parameter.
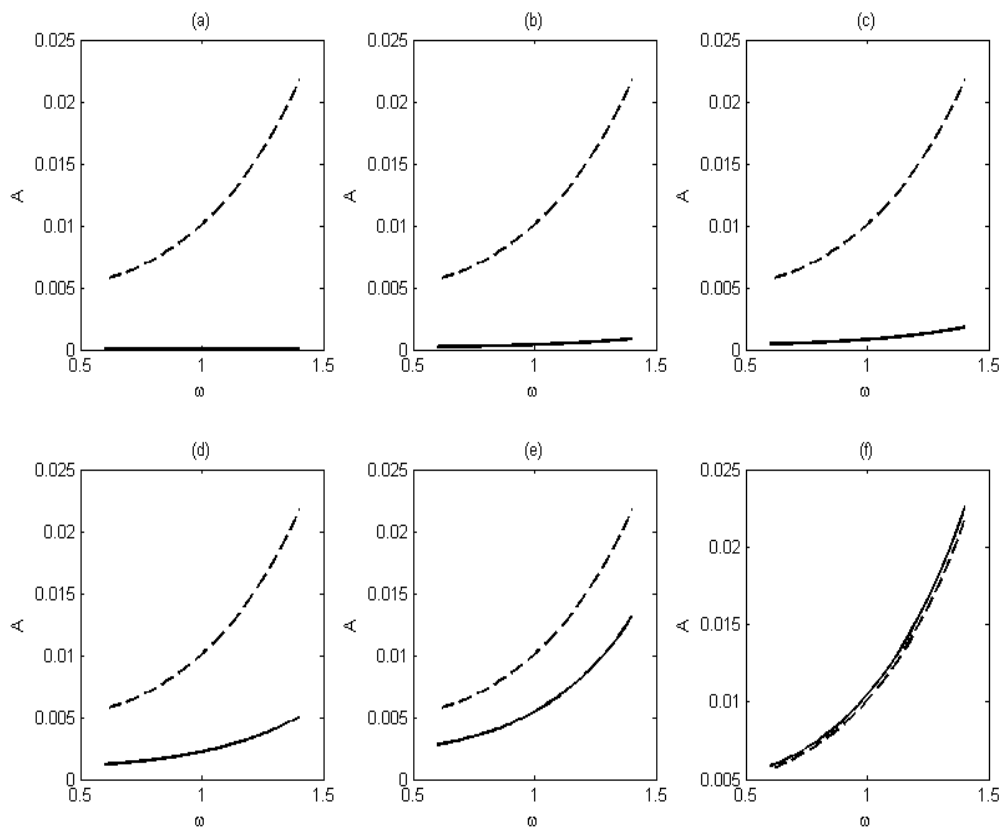

Figure 3. Critical values for AC actuation for (a) $h=10 \mathrm{~nm}$, (b) $h=20 \mathrm{~nm}$, (c) $h=100 \mathrm{~nm}$, (d) $h=500 \mathrm{~nm}$, (e) $h=1 \mu \mathrm{m}$, (f) $h=10 \mu \mathrm{m}$; classical theory ------- , nonlocal theory - -

The predicted region for the chaotic motion based on Melnikov method, Eq. (21), is shown in Грешка! Източникът на препратката не е намерен.3 for different beam thicknesses. The nonlocal parameter for this simulation is set as $e_{0} a=6 \mu \mathrm{m}$.

It can be seen that, for beam thickness values higher than $10 \mu \mathrm{m}$, the predicted regions for chaos based on classical continuum and nonlocal theories emerge and size effect is not present. By decreasing the beam thickness, the size effect becomes dominant and the harmonic region based on the nonlocal theory becomes different to the one obtained by classical continuum theory.

The nano-resonator response for $\omega=1, h=10 \mathrm{~nm}$ and $A=0.003$ is simulated using classical and nonlocal theories. Figure (a) and 4(b) show the time response and phase plane of the nano-resonator based on classical theory, respectively. As can be seen, the response of the system is chaotic, which is in agreement with Melnikov analysis.
Figure (5) shows the Poincare map of the system response based on classical theory which indicates chaotic motion.

The corresponding results for the nano-resonator based on the nonlocal theory are shown in Figure 6. It can be seen that the response of the system is not chaotic and the vibration of the nano-resonator is harmonic.

Figure (7) shows the Poincare map of the system based on the nonlocal theory which indicates harmonic motion. 

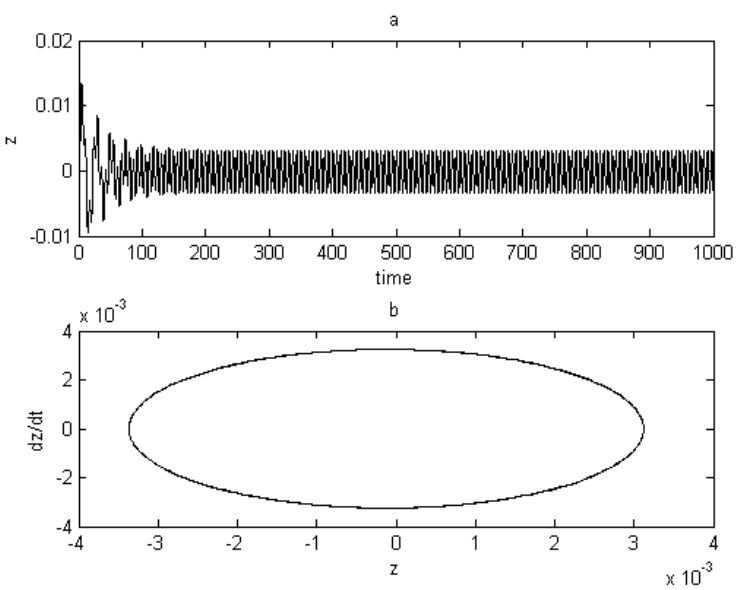

Figure 4. (a) Time response and (b) phase plane of the nanoresonator using classical continuum theory.

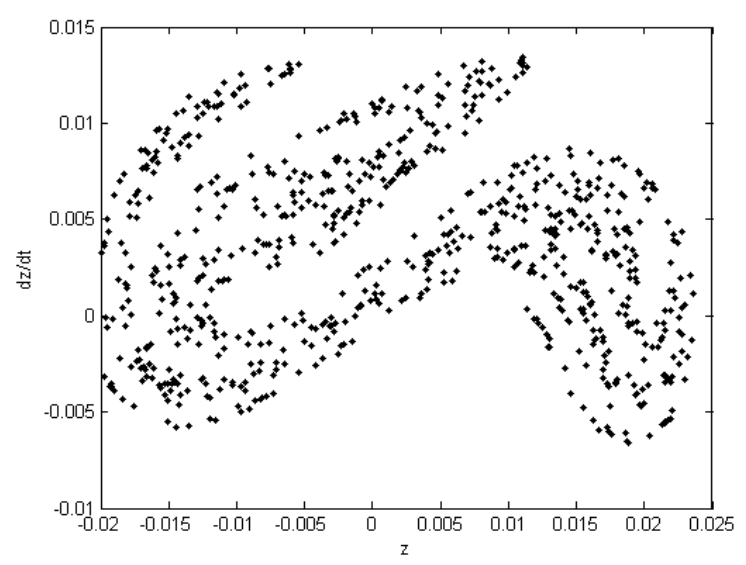

Figure 5. Poincare map of the nano-resonator based on classical theory.
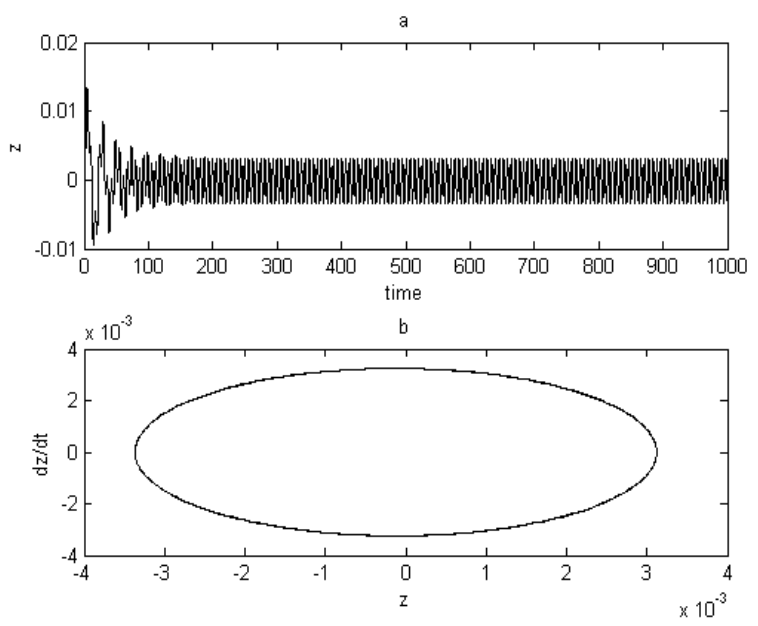

Figure 6. (a) Time response and (b) phase plane of the nanoresonator using nonlocal continuum theory.

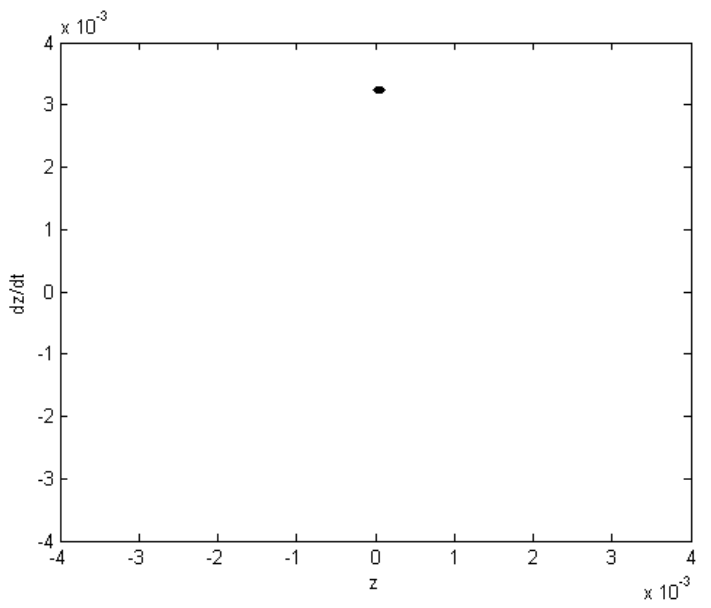

Figure 7. Poincare map of the nano-resonator system based on nonlocal theory.

\section{Conclusions}

In this paper, nonlocal elasticity theory is used to predict chaos in MEMS/NEMS resonators. In order to use Galerkin decomposition method, the mode shape of the beam based on nonlocal theory is found and used to convert the governing differential equation to ordinary one. Melnikov method is used to obtain the critical actuation amplitude leading to chaotic motion. Effects of the nonlocal parameter and the beam thickness are investigated and the following results are obtained:

- Increasing the nonlocal parameter decreases the nonlinear stiffness and increases the critical actuation voltage which may lead to chaotic motion.

- For the beams thicknesses more than 10 micrometer, size effect can be ignored.

- Decreasing the beam thickness increases the difference between the results of the two theories.

\section{References}

1. A. Tocchio, A. Caspani, G. Langfelder, Sens. J. IEEE, 12, 1719-25, (2012)

2. A.C. Luo, F.-Y. Wang, Commun. Nonl. Sci. Num. Simul., 7, 31-49, (2002)

3. H.S. Haghighi, A.H. Markazi, Commun. Nonl. Sci. Num. Simul., 15, 3091-9, (2010)

4. C. Tang, G. Alici, J. Phys. D: Appl. Phys., 44, 33550, (2011)

5. A.C. Eringen, Nonlocal continuum field theories: Springer, (2002)

6. C. Lim, C. Li, J.-L. Yu, Acta Mech. Sin., 26, 755765, (2010)

7. Y.-G. Hu, K.M. Liew, Q. Wang, X. He, B. Yakobson, J. Mech. Phys. Sol., 56, 3475-3485, (2008) 
8. M. Strozzi, L.I. Manvitch, F. Pellicano, V.V. Smirnov, D.S. Shepelev, J. Sou. Vib., 233, 2936-2957, (2014)

9 C.W. Lim, C. Li, J.L. Yu, Interact. Multis. Mech., 2, 223-233, (2009) 